\title{
TERAPIA COMPORTAMENTAL PARA INCONTINÊNCIA URINÁRIA DA MULHER IDOSA: UMA AÇÃO DO ENFERMEIRO
}

\author{
Célia Pereira Caldas ${ }^{1}$, Ivone Renor da Silva Conceição², Rita Marinela da Cruz José3 , Barbara Martins Corrêa \\ da Silva
}

${ }^{1}$ Doutora em Enfermagem. Professora Adjunta da Faculdade de Enfermagem da Universidade do Estado do Rio de Janeiro (UERJ). Rio de Janeiro, Brasil. E-mail: celpcaldas@hotmail.com

${ }^{2}$ Mestranda em Enfermagem pelo Programa de Pós-Graduação em Enfermagem da Faculdade de Enfermagem da UERJ. Chefe de Enfermagem do Núcleo de Atenção ao Idoso. Rio de Janeiro, Brasil. E-mail: renorivn@yahoo.com.br

${ }^{3}$ Especialista em Terapia Intensiva. E-mail-ritamarinela@yahoo.com.br

${ }^{4}$ Especialista em Geriatria e Gerontologia. Residente do Hospital Universitário Pedro Ernesto. E-mail: barbaramartins_enf@ yahoo.com.br

RESUMO: O objetivo deste trabalho foi relatar uma experiência de implementação da Terapia Comportamental para incontinência urinária como uma estratégia para melhorar o controle urinário e qualidade de vida da mulher idosa. Os dados foram coletados em 12 prontuários de mulheres que apresentavam diagnóstico de incontinência urinária mista ou de esforço e são apresentados descritivamente. Três pacientes obtiveram diminuição da frequência e seis pacientes zeraram as perdas. Oito pacientes referiram que os exercícios perineais foram o elemento da terapia que mais ajudou. Seis pacientes referiram que a terapia comportamental melhorou a autoestima e o autocuidado. Seis pacientes relataram que a Terapia Comportamental ajudou a lidar com o problema. Conclui-se que este procedimento deve ser valorizado como uma ação de enfermagem para a prática clínica em decorrência de sua efetividade observa.

DESCRITORES: Enfermagem. Terapia comportamental. Incontinência urinária

\section{BEHAVIORAL THERAPY FOR URINARY INCONTINENCE OF ELDERLY WOMAN: A NURSING ACTION}

\begin{abstract}
The aim of this study was presente to assess the experience of implementation of behavioral therapy for urinary incontinence as a strategy to improve bladder control and quality of life in elderly women. Data were collected from medical records of 12 women who were diagnosed with mixed or stress incontinence. Data are presented descriptively. Three patients had decreased frequency and six patients zeroed losses. Eight patients reported that perineal exercises were the part of therapy that helped most. Six patients reported that behavioral therapy improved self-esteem and self-care. Six patients reported that behavioral therapy helped them to cope with the problem. It is concluded that this procedure should be valued as a nursing action for clinical practice because of its effectiveness notes.
\end{abstract}

DESCRIPTORS: Nursing. Behaviour therapy. Urinary incontinence

\section{LA TERAPIA COMPORTAMENTAL PARA LA INCONTINENCIA URINARIA DE MUJERES ANCIANAS: UNA ACCIÓN DEL ENFERMERO}

\begin{abstract}
RESUMEN: El objetivo de este estudio fue evaluar la experiencia de la aplicación de la terapia comportamiental para la incontinencia urinaria como una estrategia para mejorar el control de la vejiga y la calidad de vida de las mujeres de edad avanzada. Los datos fueron recogidos de las historias clínicas de 12 mujeres que fueron diagnosticados con incontinencia mixta o de estrés y se presentan de manera descriptiva. Tres pacientes presentaron disminución de la frecuencia y seis pacientes a cero pérdidas. Ocho pacientes informaron de que los ejercicios perineales formaban la parte de la terapia que más las ha ayudado. Seis pacientes reportaron que la terapia comportamental mejora de la autoestima y el autocuidado. Seis pacientes reportaron que la terapia le ayudó a hacer frente al problema. Se concluye que este procedimiento debe ser valorado como una acción de enfermería para la práctica clínica debido a su eficacia observada.
\end{abstract}

DESCRIPTORES: Enfermería. Terapia conductista. Incontinencia urinaria 


\section{INTRODUÇÃO}

A Incontinência Urinária (IU) é um problema que acomete muitos idosos, principalmente as mulheres. É considerada como uma questão social, pois tem repercussões negativas sobre a qualidade de vida, higiene e atividade sexual dos pacientes. A vida social passa a depender da disponibilidade de banheiros e, comumente, eles relatam preocupação e embaraço com o odor da urina. ${ }^{1}$

A incontinência urinária pode ser classificada em três tipos principais: a Incontinência Urinária de Esforço (IUE), que ocorre quando há perda involuntária de urina durante o esforço, exercício, ao espirrar ou tossir; a Incontinência Urinária de Urgência (IUU), que é caracterizada pela queixa de perda involuntária de urina acompanhada ou precedida por urgência e; a Incontinência Urinária Mista (IUM), que ocorre quando há queixa de perda involuntária de urina associada à urgência e também aos esforços. ${ }^{2}$

Dependendo das condições de saúde, tipo e estágio da incontinência, o tratamento pode ser cirúrgico, medicamentoso, fisioterápico ou comportamental. Através destas intervenções a cura pode ser obtida, os sintomas podem ser minimizados ou a mulher pode aprender a lidar melhor com o problema.

No Brasil, a abordagem de tratamento é tradicionalmente cirúrgica. ${ }^{3}$ Entretanto, este procedimento envolve custo elevado e pode ocasionar complicações. Atualmente, tem surgido o interesse por opções de tratamentos menos invasivos. A partir desta idéia, surgiu a proposta de realizar a Terapia Comportamental (TC) em pacientes com diagnóstico de IUE e IUM em um serviço ambulatorial geriátrico de um hospital universitário. A TC implementada inclui orientações sobre hábitos de vida, promoção do auto-cuidado, diário miccional, exercícios perineais e avaliação da resposta da paciente à terapia.

Este estudo tem como objetivo relatar a experiência de implementação da TC, considerando-a como uma estratégia para melhorar o controle urinário e qualidade de vida da mulher idosa.

O propósito é agregar conhecimento e propor soluções no campo da Enfermagem para um problema que vem sendo apontado por outros pesquisadores como uma questão de Enfermagem e de Saúde Pública.

Em abril de 2010, realizamos um levantamento bibliográfico em base de dados através da Biblioteca Virtual em Saúde (BVS) nas fontes Base de dados em Enfermagem (BDENF), Literatura Latino Americana e do Caribe em Ciências da Saúde (LILACS), Literatura Internacional em Ciências da Saúde (MEDLINE), e SciELO (Scientific Eletronic Library Online) utilizando os descritores enfermagem e incontinência urinária. Foram encontrados 26 artigos na BDENF, 24 artigos na LILACS, 752 artigos no MEDLINE e 23 artigos na SCIELO.

Estes estudos abordavam a prevalência da incontinência urinária, os diagnósticos de enfermagem, a qualidade de vida das mulheres e as intervenções de enfermagem. Foram encontrados apenas quatro artigos sobre a ação do enfermeiro na TC para incontinência urinária da mulher idosa. Portanto, constata-se a relevância deste estudo, uma vez que os enfermeiros têm uma contribuição importante para resolver um problema que tem impacto negativo na qualidade de vida da população.

O grau de angústia experimentado e a amplitude das dificuldades estão relacionados tanto com a idade, etnia ou religião, quanto com a percepção que cada indivíduo tem de sua incontinência, o que levará aos diferentes níveis de transtorno emocional e pela busca (ou não) de tratamento. ${ }^{4}$ Além disso, há barreiras percebidas na manutenção do autocuidado. Sendo assim, a IU pode gerar sofrimento e as mulheres incontinentes enfrentam dificuldades para lidar com esse problema.

O serviço de saúde que é o cenário deste estudo conta com a participação do enfermeiro, integrada com a equipe médica, com cuidadores, familiares e idosos desde 1994. A intervenção de enfermagem privilegia o investimento no autocuidado para o convívio com a incontinência urinária, quando inevitável, mas atuando terapeuticamente na promoção da continência.

\section{A EXPERIÊNCIA DE IMPLEMENTAÇÃO DA TERAPIA COMPORTAMENTAL APLICADA PELO ENFERMEIRO}

A proposta de trabalhar no Ambulatório de Geriatria surgiu a partir da necessidade de ter a equipe de enfermagem como integrante da equipe multidisciplinar no atendimento a mulher idosa com incontinência urinária. Na ocasião, havia uma pesquisa sendo realizada, na qual se comparavam os resultados do tratamento medicamentoso e com a eletroestimulação transvaginal nas pacientes com incontinência urinária de urgência.

Deste modo, o ambulatório da Urogeriatria foi organizado da seguinte maneira: as pacientes 
com diagnóstico urodinâmico de incontinência urinária de urgência eram designadas para a terapia medicamentosa ou para a eletroestimulação e as pacientes com incontinência urinária de esforço e mista eram encaminhadas para a TC com o enfermeiro.

A TC envolve o planejamento de estratégias de intervenção, sua aplicação e avaliação dos resultados. A avaliação do enfermeiro parte dos aspectos fisiológicos, sem, entretanto, deixar de enfocar os aspectos psicológicos, sociais e ambientais que influenciam o problema. ${ }^{5}$

Ao realizar a anamnese o enfermeiro deve procurar saber o início, duração, freqüência, período, quantidade e características da perda de urina, devendo ser avaliados sintomas de incontinência urinária, hábitos higiênicos, alimentares e de hidratação. No Exame físico, são avaliadas lesões no assoalho pélvico associadas à incontinência, como prolapso uterino, rotura perineal, lesões irritativas e sinais flogísticos.

Outro aspecto importante é a avaliação da capacidade de autocuidado dos pacientes, história genitourinária, neurológica e clínica em geral, medicações e auto-percepção sobre a incontinência urinária. $^{5}$

Os objetivos da consulta de enfermagem na TC são: melhorar a condição de idosas incontinentes através de cuidados básicos de higiene, educação, controle miccional, prevenção de lesões no períneo e favorecer um ambiente adequado para manter a continência, evitando complicações.

O protocolo realizado para esta pesquisa tem a duração de quatro meses, com uma consulta de enfermagem a cada mês. Todas as mulheres que iniciam a TC têm como diagnóstico de enfermagem "Padrões de Eliminação Urinária Alterados" que é definido como o estado em que o indivíduo apresenta ou corre o risco de apresentar disfunção na eliminação urinária. ${ }^{6}$

Em seguida, são estabelecidas as intervenções de enfermagem a partir das orientações sobre hábitos de vida, exercícios perineais e diário miccional. Os resultados esperados, ao final da terapia, é que a idosa se mantenha continente, isto é, que ela consiga zerar as perdas urinárias e ou que seja capaz de identificar os fatores que influenciam o aumento dos sintomas de incontinência para que o seu comportamento seja ajustado a fim de que a idosa atinja uma melhora na sua qualidade de vida.
As orientações sobre hábitos de vida mais freqüentes são as seguintes: estabelecer um ritmo miccional; evitar ingestão excessiva de líquidos à noite; evitar ingestão de cafeína e álcool; diminuir a massa corporal (se necessário) e; regularizar os hábitos intestinais.?

Os exercícios perineais, conhecidos como exercícios de Kegel, consistem em contrair e relaxar a uretra com a ajuda da contração dos músculos levantadores do ânus, mantendo a contração por cinco segundos com intervalos de 10 segundos. ${ }^{8}$ Um fator importante de continência urinária é a rede de sustentação formada pelas fibras do músculo levantador do ânus ligadas à fáscia endopélvica que circundam vagina e porção distal da uretra. Durante a contração muscular as fibras irão tracionar a fáscia endopélvica em direção ao púbis e comprimí-la contra a parede vaginal, mantendo a luz uretral ocluída. ${ }^{9}$

Por fim, o diário miccional foi um instrumento utilizado para avaliar o comportamento do paciente ao longo de três dias. Através do diário podemos avaliar a ingesta de líquido, as eliminações com seus respectivos horários e medidas, identificar as perdas urinárias, por urgência, por esforço ou pelos dois, a intensidade e a quantidade dessas perdas.

A paciente recebe dois copos graduados. No primeiro ela irá medir e depois anotar no diário o quanto ingeriu de líquido. No outro copo ela irá medir e depois anotar no diário a quantidade de urina e o horário da micção. Em seguida, deverá anotar se houve perda e se foi intensa, moderada ou pouca, além de anotar a intensidade da urgência. Além destas informações a mulher é orientada a anotar se houve qualquer perda de urina por esforço, tosse ou espirro.

$\mathrm{Na} 1^{\mathrm{a}}$ consulta de enfermagem é realizada a entrega do material para a realização do diário miccional, orientações comportamentais e sobre os exercícios perineais. No mês seguinte ocorre a $2^{a}$ consulta de enfermagem, quando é realizada a avaliação do diário miccional e da evolução do quadro. No terceiro mês acontece a $3^{\mathrm{a}}$ consulta, quando é realizada a entrega do material para realização de outro diário micional. Na semana seguinte, a paciente retorna para a $4^{a}$ consulta onde é avaliado o diário miccional e este é comparado com o que foi realizado na $1^{a}$ consulta. Assim, é possível realizar a avaliação da influência da TC no controle urinário e no cotidiano da paciente ao final das quatro consultas. 


\section{MÉTODO}

Trata-se da descrição dos resultados de uma experiência, a partir de dados coletados nos prontuários de mulheres atendidas no ambulatório geriátrico de um hospital universitário.

O campo de estudo foi o ambulatório de urogeriatria de um hospital universitário. Este projeto acompanha mulheres idosas com queixas de incontinência urinária e possui um protocolo que inclui três abordagens: o tratamento medicamentoso, o fisioterápico e a TC realizada pela enfermagem.

Os dados foram coletados a partir de 12 prontuários de mulheres, cujas idades variaram entre 64 anos e 79 anos. Este quantitativo de prontuários corresponde ao número de pacientes atendidas neste projeto no período de três meses, aproximadamente.

Os critérios de inclusão foram: ser mulher, ter mais de 60 anos e apresentar diagnóstico pela urodinâmica de incontinência urinária de esforço ou mista. Foram considerados critérios de exclusão: possuir diagnóstico pela urodinâmica de incontinência de urgência, pois estas pacientes eram encaminhadas para o tratamento medicamentoso ou fisioterápico.

A coleta de dados foi realizada de acordo com os seguintes procedimentos: coleta dos dados do prontuário registrados na evolução de enfermagem e do diário miccional. Foram colhidos dados de prontuários das pacientes atendidas no período de janeiro a maio de 2008. Os dados foram coletados em junho de 2008.

A coleta de dados do diário miccional incluiu as seguintes variáveis relativas a um período de três dias: freqüência miccional, necessidade urgente de urinar e episódios de perda involuntária de urina.

A coleta dos dados do prontuário registrados na evolução de enfermagem foi realizada a partir de um roteiro elaborado para este fim, o qual possui 10 perguntas fechadas e que incluiu as seguintes variáveis: idade, tipo de incontinência urinária, queixa principal relacionada à incontinência urinária, há quanto tempo está com perda urinária, se já realizou cirurgia de períneo, impacto negativo da incontinência urinária no cotidiano da paciente, expectativa da paciente sobre a TC, maior dificuldade durante a terapia, elemento da terapia comportamental que a paciente aponta ter ajudado mais, como a TC influenciou no controle miccional da paciente.
Para a realização do estudo, foi solicitada autorização para consulta ao prontuário à Direção da Unidade e ao Comitê de Ética em Pesquisa do Hospital Universitário Pedro Ernesto (CEP/ HUPE/UERJ) $n^{\circ}$ 2005-CEP/HUPE aprovado em 20 de março de 2008.

A identificação da paciente e o registro do prontuário foram mantidos em anonimato durante todas as fases do estudo de acordo com as diretrizes e normas de pesquisa envolvendo seres humanos estabelecidos pela Resolução ${ }^{\circ} 196$ do Conselho Nacional de Saúde de 10 de Outubro de 1996. ${ }^{10}$ As participantes assinaram um Termo de Consentimento Livre Esclarecido, no qual, após esclarecidas sobre os objetivos e procedimentos da experiência, autorizaram o uso dos dados contidos em seus respectivos prontuários.

Para a descrição dos resultados da experiência foram considerados os seguintes parâmetros:

$\mathrm{Na}$ análise do diário miccional foram observadas mudanças dos parâmetros miccionais. Estas mudanças, que serviram como critério de melhora do quadro de incontinência urinária foram as seguintes: diminuição da freqüência miccional, diminuição da necessidade urgente de urinar e diminuição das perdas involuntárias de urina em relação a sua condição inicial.

Foram comparados os resultados da análise do diário miccional com a análise dos dados da evolução de enfermagem e assim, foi possível analisar a influência da TC aplicada pelo enfermeiro na mudança nos parâmetros miccionais e no cotidiano das pacientes. Deste modo, avaliaramse os benefícios da TC nas mulheres idosas com incontinência urinária.

\section{RESULTADOS}

A incontinência urinária era a queixa principal em oito mulheres. Estas mulheres apresentavam a incontinência há mais de dois anos. Destas pacientes, quatro conseguiram zerar as perdas urinárias.

Quanto à freqüência, três pacientes obtiveram diminuição, seis pacientes obtiveram diminuição da necessidade urgente de urinar e seis pacientes zeraram as perdas.

A metade das pacientes já havia realizado cirurgia de períneo. Dentre as seis pacientes que conseguiram zerar as perdas urinárias, quatro delas não fizeram cirurgia de períneo. Além disso, duas pacientes que já haviam feito as cirurgias conseguiram zerar as perdas urinárias. 
Todas as pacientes referiram que, embora a IU não as impedisse de realizar as suas atividades cotidianas, causavam desconforto e mal estar ao longo do dia.

Todas referiram que estavam com expectativas em relação à TC. Seis pacientes referiram que não houve dificuldades para colocar em prática as orientações sobre hábitos de vida, os exercícios perineais bem como na realização do diário miccional. Enquanto que quatro pacientes referiram dificuldades na realização do diário miccional.

Oito pacientes referiram que os exercícios perineais foram o elemento da terapia que mais ajudou. Seis pacientes referiram que a TC melhorou a auto-estima e o autocuidado. Seis pacientes relataram que a TC ajudou a lidar com o problema. Além disso, três pacientes referiram que a TC minimizou os sintomas.

\section{DISCUSSÃO}

Os resultados desta experiência de implementação de TC foram coerentes com a relevância que a literatura dá ao problema, pois há maior probabilidade das mulheres desenvolverem incontinência urinária à medida que envelhecem, sendo a IUE a principal causa de incontinência urinária. ${ }^{11-12}$ Entretanto, estudos afirmam que o diagnóstico correto da causa da incontinência urinária é muito importante para que seja instituída a terapêutica adequada, sendo a avaliação clínica insuficiente para o diagnóstico correto da IUE, deste modo, o estudo urodinâmico é fundamental. ${ }^{12}$

A experiência ressaltou que a TC pode ser introduzida ainda que a incontinência não esteja em fase inicial. Isso significa que com a TC é possível evitar uma intervenção cirúrgica. A grande vantagem é que a TC apresenta mínimos efeitos colaterais e não impossibilita um tratamento cirúrgico futuro. ${ }^{3}$

Observou-se também que o benefício da TC vai além da melhora dos parâmetros miccionais, pois receber informação e ser orientado por um profissional a respeito de como lidar com o seu problema é uma estratégia de promoção da saúde. O autocuidado é a prática de atividades iniciadas e executadas pelos indivíduos em seu próprio benefício para manutenção da vida, da saúde e do bem estar. ${ }^{13}$ Deste modo, o idoso junto ao profissional de enfermagem, torna-se co-participante e comprometido com o processo de busca para identificar as suas dificuldades em realizar os próprios cuidados e encontrar resolução. Assim, a enfermagem assiste o ser humano no atendimento de suas necessidades básicas pelo ensino do autocuidado para manter e promover a saúde. ${ }^{15}$

\section{CONCLUSÃO}

Este estudo relatou uma experiência bem sucedida de aplicação de uma ação de enfermagem. Sua limitação foi o pequeno número de casos avaliados. No entanto, temos a destacar que seu objetivo principal foi alcançado. Recomenda-se a realização de estudos de intervenção para verificação da efetividade desta ação ao longo do tempo e com uma amostra de pacientes maior.

Os resultados apontam para o valor desta ação de enfermagem para a prática clínica. A TC deve ser aplicada antes de encaminhar uma paciente com incontinência urinária para realizar um procedimento cirúrgico, pois é possível obter uma melhora do quadro. Além disso, mesmo quando houver necessidade da intervenção cirúrgica, a TC pode ser realizada após tal procedimento a fim de otimizar a reabilitação no pós-operatório tardio.

Sugerimos para os próximos estudos a padronização das ações de enfermagem incluídas neste procedimento, a fim de permitir a comparação dos resultados obtidos no presente trabalho com futuros estudos.

\section{REFERÊNCIAS}

1. Fonseca ESM, Camargo ALM, Castro RA, Sartori MGF, Fonseca MCM, Lima GR, et al.Validação do questionário de qualidade de vida (King`s Health Questionnaire) em mulheres brasileiras. Rev Bras Ginecol Obstet. 2005 Mai; 27(5):235-42.

2. Borba AMC, Lelis MACS, Bretas, ACP. Significado de ter incontinência e ser incontinente na visão das mulheres. Texto Contexto Enferm [online]. 2008 Ago-Out [acesso 2010 Abr 11]; 17(3): Disponível em http:/ / www.scielo.br/scielo.php?script=sci_arttex t\&pid=S0104-07072008000300014

3. Rett MT, Simões JA, Herman V, Gurgel MSC, Morais SS. Qualidade de vida em mulheres após o tratamento da incontinência urinária de esforço com fisioterapia. Rev Bras Ginecol Obstet. 2007 Ago; 29(3):135-40.

4. Higa R, Lopes MHBM, Turato ER. Significados psicoculturais da incontinência urinária feminina: uma revisão. Rev Latino-am Enfermagem. 2008. Ago; 16(4):779-86.

5. Rodrigues RAP, Mendes MMR. Incontinência Urinária em idosos: proposta para conduta da enfermeira. Rev Latino-am Enfermagem. 1994 Jul; 2(2):5-20. 
6. Carpenito LJ. Manual de diagnóstico de enfermagem. $11^{\mathrm{a}}$ ed. Porto Alegre (RS): Artes Médicas; 2008

7. Wei J, Raz S, Young GPH. Fisiopatologia da Incontinência Urinária de Esforço. In: Rubinstein I, organizador. Urologia Feminina. São Paulo (SP): BYK; 1999. p. 167-78.

8. Brunner LS. Tratamento de pacientes com distúrbios urinários e renais. In: Smeltzer S, Bare B, organizadoras. Brunner \& Suddarth: Tratado de enfermagem médico-cirúrgica. $9^{\mathrm{a}}$ ed. Rio de Janeiro (RJ): Guanabara Koogan; 2002.v.3. p.1100-22.

9. Matheus LM, Mazzari CF, Mesquita RA, Oliveira J. Influência dos exercícios perineais e dos cones vaginais, associados à correção postural, no tratamento da incontinência urinária feminina. Rev Bras Fisioterapia. 2006 Dez; 10(4):387-92.

10. Ministério da Saúde (BR), Conselho Nacional de Saúde, Comissão Nacional de Ética em Pesquisa. Resolução n 196 de 10 de outubro de 1996:diretrizes e normas regulamentadoras de pesquisa envolvendo seres humanos. Brasília (DF): MS; 1996.
11. Higa R, Lopes MHBM. Fatores associados com a incontinência urinária na mulher. Rev Bras Enferm. 2005 Jul-Ago; 58(4):422-8.

12. Silva JAL, Santos JLS, Camargos AF. O valor da perda de urina pelo óstio uretral externo, detectada pelo exame físico, em mulheres com queixa de perda urinária apenas aos esforços, no diagnóstico urodinâmico de incontinência urinária de esforço pura. J Bras Ginec 1995 Abr; 105(4):159-62.

13. Geoge JB. Teorias de enfermagem. Porto Alegre (RS): Artes Médicas; 1993.

14. Lenardt MH, Hammerschimidt KSA, Borghi ACS,Vaccari E, Seima MD. O idoso portador de nefropatia diabética e o cuidado de si. Texto Contexto Enferm [online]. 2008 Jun-Jul [acesso 2010 Abr 29]. 17(2): Disponível http://www.scielo.br/ scielo.php?script=sci_arttext\&pid=S0104-0707200 8000200013

15. Horta WA. Processo de enfermagem. São Paulo (SP): EPU Ed. da Universidade de São Paulo; 1979. 